$\xi=-$ 国

\title{
License Plate Recognition System Using Matlab GUI For Surveillance
}

\author{
M. Venkata Srinu*, Venkateswara Rao Morla, Kali Vara Prasad Baditha, Vara Kumari. S, Srinivas Maddimsetti \\ Department of Electronics and Communication Engineering, Koneru Lakshmaiah Education Foundation, \\ Vaddeswaram, Guntur, Andhra Pradesh, India -522502 \\ *Corresponding author E-mail: venkat486@kluniversity.in
}

\begin{abstract}
License plate recognition is an essential task in applications like urban vehicle management, intelligent transportation system, traffic surveillance and parking management system. In this work, we acquire the images using the mobile app and recognize license plate details with the help of our proposed image processing model. The recognized license plate details have been displayed on a customized website. The proposed image processing model does the adaptive thresholding on the images with resolution of $1280 \times 960$. The connected component analysis using bounding boxes will be performed on threshold image. The desired plate details are highlighted by creating a region of interest for maximum magnitude row of an image based on the pixel value, then the statistical and logical operations are used to extract the candidate region. After obtaining candidate region, recognition of license plate number has been done using template matching. The complete customer details, displayed on the customized website which is connected to the database with the help of plate number. The computation time of proposed method is less than the existed methods.
\end{abstract}

Keywords: adaptive thresholding, connected component analysis (CCA), template matching

\section{Introduction}

The increase in the number of vehicles from the past few decades led to the development of license plate detection methods to reduce the human intervention. Many detection methods were developed in various countries which were successfully implemented from the year 1975. But due to development of the technology, many methods

took its root in developing more accurate detection. License Plate Recognition is an image processing technology which is used to identify the vehicles and details by extracting and recognizing their license plate number. The License Plate number is used to extract more information about the vehicle and it's owner details which is used for further processing. For License plate recognition, Gaussian filtering and morphological operations give efficient results if the number plate follows regular pattern [1]. The Scale-Invariant Feature Transform (SIFT) and template matching technique also not giving efficient results while the low resolution and blurred image feed to the system [2]. There are numerous vehicle tracking systems and many of these tracking systems use highly sophisticated web cameras [3] for capturing images. This resulted in the cost increment of both hardware and software equipment. Many of these tracking systems were developed for transportation system applications. The automatic number plate recognition systems suffer from various constraints which uses more resources in terms of hardware, fixed distance, and detection rate. An improvement in the vehicle License plate Detection such as reduced computation time, less complexity and detecting License Plate region more accurately with fewer cost systems will make it a more real-time detection system than the existing models. The background and plate sizes vary from one country to the other, issues can be overcome by doing perfect template matching. The system based on pulse coupled neural networks and template matching was unable to perform well because that is incapable to convert text or data accurately [4]. The unwanted line elimination method, chin code, radon transform and multilayer perceptron are giving 80In our proposed License Plate Detection Method, a mobile camera with $1280 \times 960$ resolution is used instead

of web cameras to capture the License plate (LP) images. An online streaming process is performed to transmit the video and detect the plate details. The major steps involved in this proposed method are adaptive thresholding, connected component analysis, size filtering and finally selecting the true plate regions by maximum row size magnitude and extracting it to the text file. All these steps are performed using MATLAB software. MATLAB is the better choice for implementing License Plate Detection and Recognition System and for detecting the errors efficiently before the proposed method is actually implemented on the real time. And we are using VISUALSTUDIO for the creation of a website to automatically display the details of the vehicle.

\section{Proposed System}

The proposed system consists of two phases namely 1 . Image acquisition stage 2. Image processing method and 3. Extracting vehicle details from the server using customized website. The image acquisition has been done using mobile which will be fed to GUI, it gives extracted number to the website. The customized website drags the

vehicle details from database and display the details. The block diagram consists of three phases which are shown in Fig.1.

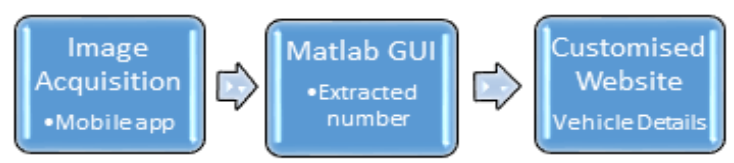

Figure 1: Block diagram of Proposed System 
The flow chart of proposed image processing method consists of step by step process as shown in figure.2. Using a Matlab customized Graphical User Interface (GUI) application which is linked to call back functions for a process the acquired image. Processing steps include grayscale conversion, adaptive thresholding, and morphological operations, connected component analysis and size filtering are performed. Template matching is done to recognize the number in the image and that will be extracted as a text file. These steps that are carried out in different stages, as depicted in Fig. 2, will be presented in the following subsections.

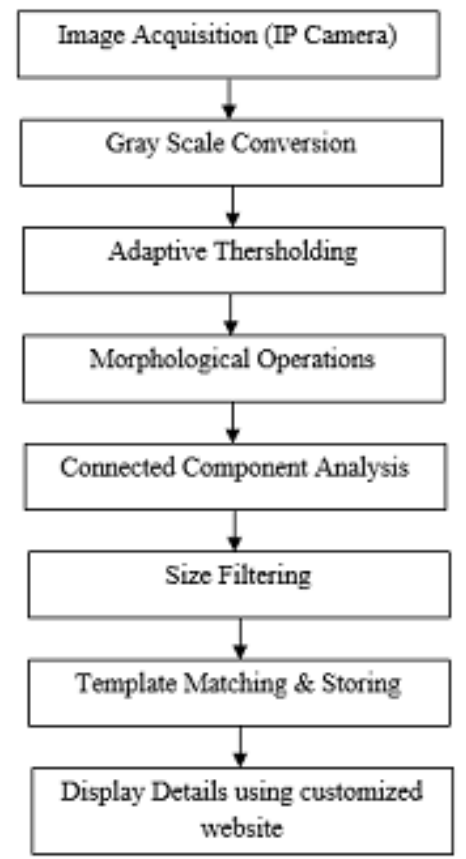

Figure 2: Flowchart of proposed method

\subsection{Image acquisition}

Image acquisition plays a major role in this system because it deals with the total cost of the system. In the proposed method, we use smartphone mobile with Android operating system and camera of photo resolution 1280x960 and video resolution of $720 \times 480$. The mobile camera transmits the live video stream from the remote location using an android application called IPWEBCAM. The advantage of this type of image acquisition is the reduction in cost. The major requirement of this type of video transmission is both computer and mobile on the same network. The screenshot of the IP WEBCAM is shown Fig.3

\subsection{Color to Gray Conversion}

The acquired color image from the mobile camera has to be converted to grayscale to reduce the image size and for faster computation. Color image in RGB (red, green, blue) color model is converted into grayscale based on the below equation

$I c=0: 299 * R+0: 587 * G+0: 114 * B$

The obtained gray scale image is fed to the adaptive thresholding stage.

\subsection{Adaptive Thresholding}

Local adaptive thresholding deals with the individual thresholding for each pixel depend on the range of intensities in its neighborhood. Window size can affect the enhancement of the binarized image. If the window is less then it results in poor thresholding and if window is large then it is more adversely affected by the illumination gradient. Hence an intermediate value is selected as window size as this window size acts as neighborhood of each pixel to check mean or median of the region and assigns the value to the pixel. For this system, we select a window of size $50 \mathrm{X} 50$ and opted for the mean. Each pixel is assigned values based on the mean value of all pixels in the selected window size. The Fig. 4 shows the acquired image and image, after the adaptive thresholding operation.

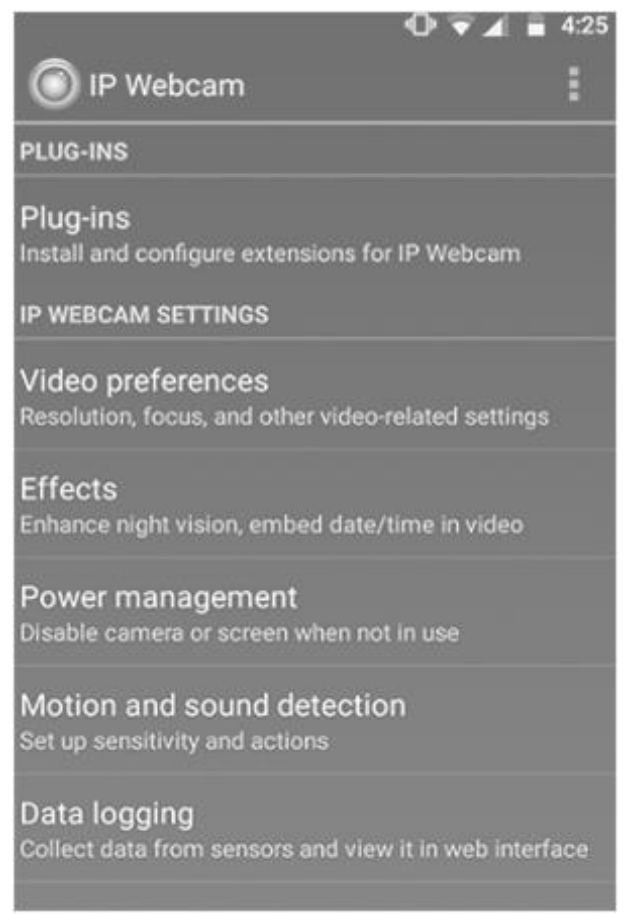

Figure 3: Screen shot of IP WEBCAM layout
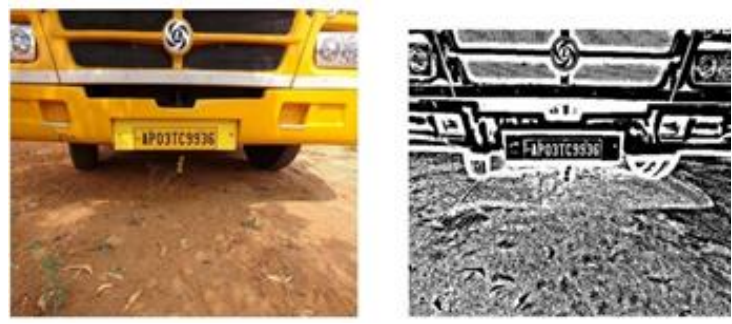

Figure 4: The acquired image and adaptive threshold image

\subsection{Morphological operations}

Pattern recognition systems use morphological operations such as dilation and erosion. These tools are used to reduce noisy objects and preserves the objects to represent targeted patterns. After adaptive thresholding, the candidate region undergoes with the dilation operation to connect broken symbols. On the other hand, the erosion is applied to filter out thinner objects in the candidate region. Here, these two operations are performed using a 1-pixeldisk element. The output of morphological stage is further processed using connected component analysis.

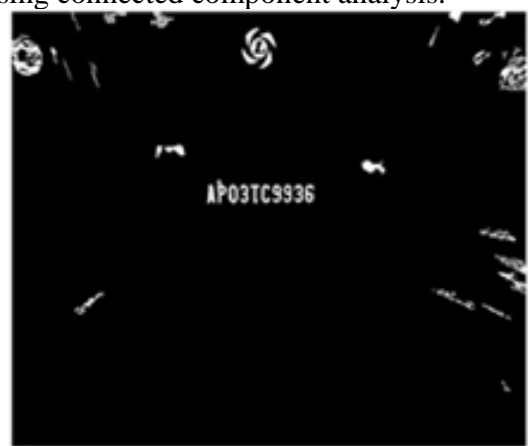

Figure 5: Morphological output image 


\subsection{Connected Component Analysis}

Connected component analysis (CCA) is very popular and familiar technique to group pixels in a labelled components depends on pixel connectivity [11-12]. An 8-point CCA will extract and locate objects for size filtering from the image which is obtained in the previous stage. The output of this stage is an array of $\mathrm{N}$ objects. All the objects that are connected are labeled and indexed. In bounding box method, it encloses the labeled region with rectangular box completely. Each object in the image is compared and indexed and by using region properties, the bounding boxes for the connected objects are giving better results.

\subsection{Size Filtering}

The objects extracted from the CCA stage are filtered based on their widths $X o b j$ and heights $Y o b j$ such that the dimensions of the License Plate symbols lie between their respective thresholds as follows:

$X \min \leq X$ ob $j \leq X \max$

$Y \min \leq Y o b j \leq Y \max$

Where $Y$ min and $X \min$ are the values below which a symbol cannot be recognized ( 8 pixels can be taken) and $X \max$ can be set to the image width divided by the number of symbols in the license number. $Y \max$ is calculated as $X \max$ divided by the aspect ratio of the used font. The range of these values can be narrowed in the case of amounted camera to speed up the process of detection but for a moving camera, this constraint depends on the required objectcamera distance. The output of this stage is an array of $\mathrm{M}$ objects. In this method we have filtered images having width less than 100 pixels and greater than 8 pixels, similarly heights greater than 8 pixels and less than 100 pixels. The size filtering stage is followed by text

region extraction which is called as candidate region extraction.

\subsection{Candidate region Extraction}

Segmentation of the image from the size filtering stage is performed to separate license number region. The filtered image is scanned row-wise from top to bottom and magnitude of the binary image in each row is stored in variables. The row having maximum magnitude will be having the license number region so that region is cropped.

The output of the cropped image is as shown in Fig. 6. The objects in the cropped image is matched with templates.

\section{AP03TC9936}

Figure 6: candidate region extraction

\subsection{Template Matching}

Template matching is one of the most famous character recognition technique present to identify the characters present in the image. A predefined set of alphabets and characters are collected and stored in the database. Each image template is created in the Matlab space and stored as variables with a size of $24 \mathrm{X} 24$. After creation of templates, the objects obtained from previous stages are correlated with the templates and the correlated values are stored in the variables. The characters for which maximum co-relation occurs is assigned with the variable. The recognized numbers are stored in the text file for further applications. Some of the templates used are shown below

in Fig. 7. The output recognized in the form of text file is opened automatically by the Matlab and stored in the directory.

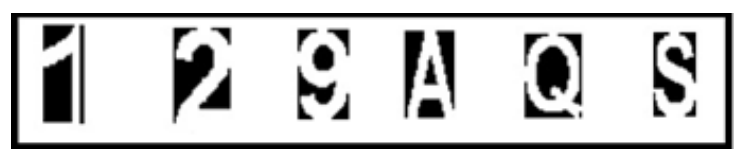

Figure 7: Sampled Templates

\section{Display Through website}

The text file from the last stage with recognized number details are fed to the customized website and performs query operations with the database file linked to it. The corresponding vehicle and owner details which are already stored in the database is displayed in the grid view of the website. All these operations are monitored through MATLAB GUI. The license number and its information should be displayed for the surveillance purpose, hence a simple website is created using $\mathrm{C}$ and DOT NET. For this purpose, we have taken development environment VISUAL STUDIO. A SQL database file is created in the VISUAL STUDIO with all collected details of the vehicle license numbers and the owner details with entry time is stored in the SQL database file. This database file is linked and configured to the website created using DOT NET and C. This customized website is automatically displays the details of the number and timing detail based on the license plate number given to it.

\section{Implementation of GUI}

All the processing and controlling operations of the license plate recognition and image acquisition with displaying the number details are controlled by using MATLAB GUI. The created model has mainly four link buttons to control the operations of the system. Push Button1 is used to access and open the mobile camera video in the browser. Push Button 2 is used to take the photo of vehicle directly from live video stream and store it in the Matlab directory. Push Button 3 is used to perform all image processing operations to extract the number and Push Button 4 links with the customized website. The GUI layout created for this work is as shown below in the Fig. 8

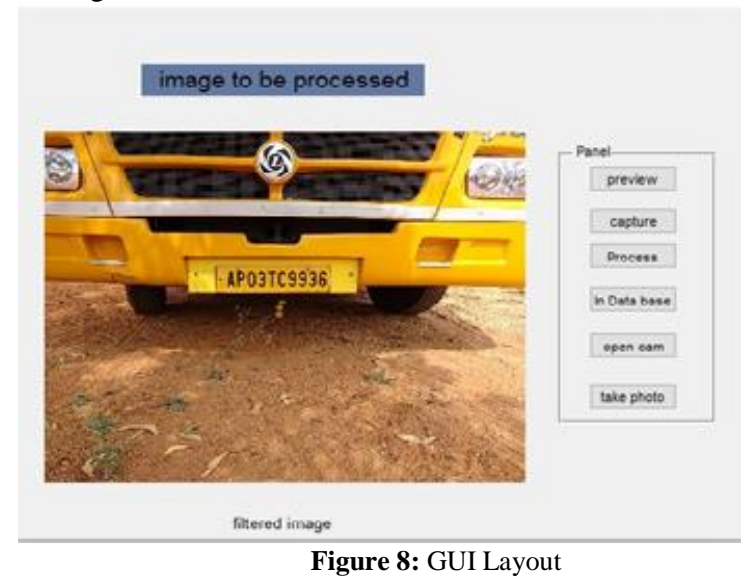

\section{Testing and Validation}

The proposed system is validated for surveillance purpose and tested for all vehicles irrespective of their colors and sizes. This method is valid up to 5-6 feet's of distance between the camera and license plate. The environmental conditions has been list out in the Table 1 to perform and validate the proposed system. Proposed method have been tested for 1200 vehicles and results shows $96.6 \%$ accuracy with good precision. The computation time for the proposed system is about $35 \mathrm{~ms}$ and this shows superiority of the method compared to existing methods. 
Table 1: List of Environment conditions

\begin{tabular}{|c|c|}
\hline Environmental conditions & Rainy, shady, sunny days \\
\hline Time & $6 \mathrm{am}-10 \mathrm{pm}$ \\
\hline License plates & 1200 images \\
\hline Camera aperture & 2.6 \\
\hline Camera distance to LP & $5-6$ feet's \\
\hline Focal length & $3.5 \mathrm{~mm}$ \\
\hline Size of image & $1280 \mathrm{x} 960$ \\
\hline Image background & Complex \\
\hline License plate sizes & Different \\
\hline License plate designs & Different \\
\hline Color of vehicles & Various \\
\hline
\end{tabular}

Table 2 represents comparison of different methods in terms of recognition accuracy and computation time. The proposed system reduces the cost and human intervention in the authorised parking systems. It can also be used in the applications such as Intelligent Transportation Systems, vehicle tracking systems and etc.

Table 2: Comparison of results

\begin{tabular}{|c|c|c|}
\hline Method & Recognition accuracy & Time (ms) \\
\hline Ref [12] & $91.94 \%$ & 200 \\
\hline Ref [13] & $93.54 \%$ & 288 \\
\hline Ref [15] & $86 \%$ & 276 \\
\hline Ref [16] & $99 \%$ & 125 \\
\hline Ref [17] & $93.1 \%$ & 223 \\
\hline Ref [18] & $93.6 \%$ & 594 \\
\hline Proposed System & $96.6 \%$ & 35 \\
\hline
\end{tabular}

\section{Conclusion}

A new license plate recognition system is proposed to recognize and access the vehicle data for the surveillance purpose. In this approach, mobile and Matlab GUI has been used which are cost effective. The image processing model is used here consists of adaptive thresholding, connected component analysis and template matching which helps the system to reduce computation time inherently. The computation time has been drastically reduced when we compare with the previous methods, the proposed system gives less computation time and high accuracy. Existing methods takes only one pixel for reference but this method takes all surrounding pixels of image while calculating adaptive thresholding and template matching. This is tested for authorized parking systems for about 1200 vehicle data and successfully achieved the results of about $96.6 \%$ accuracy with a computation time of $35 \mathrm{~ms}$.

\section{References}

[1] Rajput, H., T. Som, and S. Kar. , "An Automated Vehicle License Plate Recognition System”, Computer, 48: 56-61. 2015 doi: 10.1109/MC.2015.244

[2] H. S. Ng, Y. H. Tay, K. M. Liang, H. Mokayed, and H. W. Hon "Detection and Recognition of Malaysian Special License Plate Based on SIFT Features", CoRR, abs/1504.06921, 2015. http://arxiv.org/abs/1504.06921

[3] Naito, T., T. Tsukada, K. Yamada, K. Kozuka, and S. Yamamoto,"Robust license-plate recognition method for passing vehiclesunder outside environment", IEEE Transactions on Vehicular Technology, 49: 2309-19, 2000.

[4] W. Xiao-hua, Y. Juan-juan, M. Zhong-hua, and S. Yang"License plate recognition based on pulse coupled neural networks and template matching", Control Conference (CCC), 2014 33rd Chinese, pp. 5086-5090, 2014

[5] R. Azad and M. Nazari“"Novel and Automatic Parking Inventory System Based on Pattern Recognition and Directional Chain Code", CoRR,abs/1407.6321

2014 http://arxiv.org/abs/1407.6321

[6] A. M. AI-Ghaili, S. Mashohor, A. R. Ramli, and A. Ismail"Verticaledge-based car-license-plate detection method",
IEEE Transactions on Vehicular Technology,vol. 62, pp. 26-38, 2013.

[7] M.VenkataSrinu, G.Nagaswetha, M.Deepthi, "A multi-scale image enhancement model using human visual system characteristics", I.J. Image, Graphics and Signal Processing, DOI: 10.5815/ijigsp.2015.06.016, 1-9,May 2015

[8] V. Mai, D. Miao, and R. Wang,"Vietnam license plate recognition system based on edge detection and neural networks", Journal of information and Computing Science,vol. 8, pp. 27-40, 2013.

[9] 1. Yang, B. Hu, 1. Yu, 1. An, and G. Xiong,"A license plate recognition system based on machine vision", IEEE international Conference on Service Operations and Logistics, and informatics (SOLI) ,pp. 259-263, 2013.

[10] G. Lekhana and R. Srikantaswamy,"Real time license plate recognition system", International Journal of Advanced Technology and Engineering Research ,vol. 2, pp. 5-9,2012.

[11] F.Poriki and O.Tuzel ,"Fast construction of covariance matrices for orbirtary size image windows", International Conference on Image Processing,vol. 2, pp. 5-9,2012.

[12] Wen, Y.Lu,J.Yan,Z.Zhou,K.M.von Deneen,and P.Shi ,“An algorithm for license plate recognition applied to intelligent transportation system", IEEE Trans. Intell. Transp. Systems,vol. 12, no. 3, pp. 830-845, Sep. 2011.

[13] S. Ghofrani and M. Rasouli ,"Farsi license plate detection and recognition based on characters features", Majlesi Journal of Electrical Engineering, 5(2),vol. 12, no. 3, Vol 5 No 2 , June, 2011.

[14] Wen,Y.Lu,J.Yan,Z.Zhou,K.M.vonDeneen,andP.Shi ,"An algorithm for license plate recognition applied to intelligent transportation system”, IEEE Trans. Intell. Transp. Syst., ,vol. 12, no. 3, pp. 830-845, Sep.2011

[15] C. N. E. Anagnostopoulos, I. E. Anagnostopoulos, V. Loumos, and E. Kayafas,“A license plate-recognition algorithm for intelligent transportation system applications", IEEE Trans. Intell. Transp. Syst., ,vol. 7, no. 3, pp. 377-392, Sep. 2006.

[16] J. Jiao, Q. Ye, and Q. Huang,"A configurable method for multistyle license plate recognition", Pattern Recognition,vol. 42, no. 3, pp.358-369, 2009.

[17] Z.-X. Chen, C.-Y. Liu, F.-L. Chang, and G.-Y. Wang, “Automatic licenseplate location and recognition based on feature salience", IEEE Trans. Veh. Technol,vol. 58, no. 7, pp. 3781-3785, Sep. 2009 .

[18] J.GuoandY.Liu,"License plate localization and character segmentation with feedback self-learning and hybrid binarization techniques", IEEE Trans. Veh. Technol., ,vol. 57, no. 3, pp. 14171424, May 2008. 